\title{
Estudio genético de una familia chilena con tres fenotipos dentales diferentes
}

\author{
Rosa Andrea Pardo V1,2, Silvia Castillo $\mathrm{T}^{2}$, \\ Alexandre R Vieira ${ }^{3 a}$. \\ Genetic studies of a Chilean family \\ with three different dental anomalies
}

Background: Congenital dental anomalies can affect up to $25 \%$ of the population. Aim: To report the genetic study of a family with dental anomalies. Material and methods: We studied a Chilean family presenting with three independent dental phenotypes: third molar agenesis, supernumerary teeth, and dentinal dysplasia type I. We searched for mutations in candidate genes proposed for tooth agenesis and supernumerary teeth: IRF6, FGFR1, MSX1, MSX2, PAX9, PRDM16 and TGFA. We also studied DSPP as a candidate gene for dentinal dysplasia type I. Results: We did not find mutations in FGFR1, MSX2, PAX9, PRDM16, or TGFA. We found a MSX1 mutation (G16D) in both affected and unaffected family members. Also, we found a genetic variation not described before in IRF6 in the dentinal dysplasia type I case. Conclusions: Further investigation is necessary to evaluate if these variants are functional in nature. Finally, we are reporting a mutation in DSPP in an asymptomatic 2-year-old child, which illustrates the ethical pitfalls of interpreting molecular data for genetic counseling of young and/or asymtomatic individuals (Rev Méd Chile 2006; 134: 1541-8).

(Key words: DSPP protein, human; Genes, developmental; IRF6 protein, human; MSX1 protein, human)

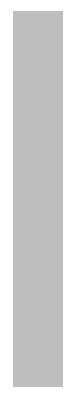

Recibido el 23 de marzo, 2006. Aceptado el 30 de mayo, 2006.

Rosa A. Pardo V. fue financiada por el Grant D43 TW-05503 del National Institutes of Health (NIH) de Estados Unidos de Norteamérica, sin que el NIH tuviera intervención en el diseño del estudio, la recolección de datos, el análisis o la interpretación de los mismos, tampoco participó dicha institución en la preparación, revisión o aprobación de este manuscrito.

${ }^{1}$ Unidad de Genética, Hospital Dr. Sótero del Río, Santiago, Chile. ${ }^{2}$ Sección Genética y Unidad de Cuidados Especiales Neonatales, Hospital Clínico Universidad de Chile, Santiago, Chile. ${ }^{3}$ Department of Oral Biology, Center for Dental and Craniofacial Genetics, School of Dental Medicine, University of Pittsburgh, Pittsburgh, PA, USA.

${ }^{\mathrm{a} O d o n t o ́ l o g o, ~ P h D ~ G e n e ́ t i c a ~}$

$\overline{\text { Correspondencia a: Alexandre R. Vieira. } 614 \text { Salk Hall. }}$ School of Dental Medicine. University of Pittsburgh. 3501 Terrace Street. Pittsburgh PA, 15261 USA. Phone \# 412 3838972. Fax \# 412 624-6685. E mail: arv11@dental.pitt.edu 
$E^{1}$ desarrollo de los dientes, folículos pilosos y glándulas mamarias es controlado por interacciones específicas entre el epitelio y los componentes mesenquimales derivados de la cresta neural. Las interacciones epitelio-mesénquima son recíprocas y secuenciales, dentro de las cuales cada componente juega un rol importante en la organogénesis.

Más de 200 genes han sido implicados en la odontogénesis, algunos de ellos codifican factores de transcripción, otros factores de crecimiento, 0 moléculas de la matriz extracelular ${ }^{1}$.

Las malformaciones dentales ocurren frecuentemente en la población, de ellas la más frecuente es la ausencia de piezas dentarias, también conocida como agenesia dentaria. La agenesia dentaria ocurre en $2 \%$ a $10 \%$ de la población (excluyendo los terceros molares, los cuales se encuentran ausentes hasta en $25 \%$ de la población). Los dientes que faltan más frecuentemente son los segundos premolares, seguidos de los incisivos laterales superiores ${ }^{2}$.

Otra alteración congénita numérica dental es la existencia de dientes supernumerarios. Los dientes supernumerarios o hiperdoncia tienen una incidencia que oscila entre $0,1 \%$ y $3,8 \%$, de acuerdo a la población que se estudie, correspondiendo un tercio de los casos a mesiodens (un diente cónico entre los incisivos centrales maxilares). La etiología de los dientes supernumerarios no está clara aún, sin embargo, hallazgos como una mayor incidencia en familiares de primer grado y un alto grado de concordancia en gemelos monocigóticos, plantean una determinación genética para esta condición ${ }^{3,4}$.

Las malformaciones dentarias también pueden afectar la mineralización de la dentina. Dicha mineralización es iniciada en la interfase de predentina a dentina, es regulada por los odontoblastos y se produce de manera frontal, caracterizada por la presencia de múltiples focos de mineralización globular (calcosferitas) que van creciendo y fusionándose con los adyacentes hasta formar un frente de mineralización relativamente uniforme.

Dentro de las patologías malformativas de la dentina se destacan dos grupos: dentinogénesis imperfecta (DI) y displasia de dentina (DD).

La dentinogénesis imperfecta se caracteriza por comprometer las dos denticiones con dientes opalescentes o ámbar, con cámaras pulpares obliteradas por dentina anormal y mayor tendencia a fracturas del esmalte. El defecto estructural de la dentina causa el daño del esmalte, la aparición de fracturas y el posterior deterioro dental. Recientemente, se han descrito mutaciones en el gen DSPP (disialofosfoproteína) relacionadas con esta enfermedad ${ }^{5,6}$ y pruebas experimentales han demostrado que mutaciones en el gen DSPP producen alteraciones en la disposición y conducen a una biomineralización defectuosa de la dentina ${ }^{7}$

Por otra parte, la displasia de la dentina es una patología poco frecuente que tiene dos subtipos: la tipo I (o radicular) y la tipo II (o coronal).

La displasia de dentina tipo I, es una enfermedad autosómica dominante, se caracteriza por tener comprometidas tanto la primera como la segunda dentición, el color de los dientes es normal, pero éstos son móviles, hay odontalgia y formación espontánea de quistes o abscesos periodontales y se asocia a pérdida dental precoz secundaria a una inadecuada formación de las raíces dentarias. A la microscopia se evidencia que la dentina de la corona es normal, pero la cámara pulpar se observa obliterada en la dentición primaria y reducida significativamente en la dentición secundaria. Diferentes teorías se han postulado para la etiopatogenia de la enfermedad, la primera es que la papila dentiniana es la responsable del cuadro en la medida que existan múltiples focos de degeneración de la misma que se calcifican y en su período de cicatrización obliteran el espacio pulpar; la segunda dice que muy tempranamente en el proceso embrionario se produce una invaginación del epitelio, lo cual genera posteriormente múltiples focos de formación de dentina; la tercera hace referencia a que existe una alteración en la interacción normal que existe entre la capa de los odontoblastos y la de los ameloblastos y que como resultado de ello se produciría una diferenciación anormal de los odontoblastos; sin que ninguna de ellas haya sido corroborada ${ }^{8}$.

Sobre la displasia de dentina tipo II, es importante recordar que su compromiso es principalmente coronal y que ha sido descrito un mapeamiento de esta patología en el cromosoma 4q21.3, en la región en la cual se encuentra el gen DSPP entre otros genes relacionados con la odontogénesis ${ }^{9}$. 
En este estudio analizamos una familia chilena con tres fenotipos dentarios diferentes: agenesia de terceros molares superiores, dientes supernumerarios y displasia de dentina tipo I, en búsqueda de mutaciones responsables de estas manifestaciones dentro de los genes ya descritos en este espectro de anomalías. Es un estudio pionero de mutaciones a nivel de genes implicados en la odontogénesis en la población chilena.

\section{MateRIAL Y MÉTODO}

Familia. Se diseñó un protocolo que fue sometido al Comité de Ética del Hospital Clínico Universidad de Chile, dentro del cual se incluyó un consentimiento informado para acceder a la participación de cada sujeto en el estudio.

A cada persona participante del proyecto, además de practicársele una evaluación clínica, le fueron tomadas radiografías panorámicas dentales de acuerdo a las especificaciones convencionales para este procedimiento, para aclarar su fenotipo dental.

En la Figura 1 se observa la genealogía con las manifestaciones clínicas y aquella información relacionada a malformaciones dentales obtenida de la evaluación radiológica.

Posteriormente se obtuvo, de todos y cada uno de los integrantes de la I, II y III generación de la familia estudiada, una muestra de $3 \mathrm{ml}$ de sangre periférica o una muestra de células en descamación de la mucosa oral tomada con un hisopo especialmente diseñado para ello (Cytosoft Brush CP-5B).

El ADN de las muestras de sangre fue extraído en la Sección Genética del Hospital Clínico Universidad de Chile y el ADN de las muestras de mucosa oral fue extraído en el Craniofacial Anomalies Research Center, University of Iowa.

Se eligieron genes candidatos basándose en datos previos de desequilibrio de ligamiento, es decir, la ocurrencia más allá de lo esperado de la asociación entre un rasgo clínico y la presencia de un segmento de ADN, enfermedades monogénicas en las cuales las anomalías dentarias forman parte de su fenotipo, patrones de expresión y modelos animales (Tabla 1).

Análisis de laboratorio. Se utilizaron PCR en volúmenes de 10-25 $\mu$ l que contenían $20 \mathrm{ng} \mathrm{ADN/}$ $\mu \mathrm{l} ; 200 \mu \mathrm{l}$ de cada nucleótido marcado (dATP,

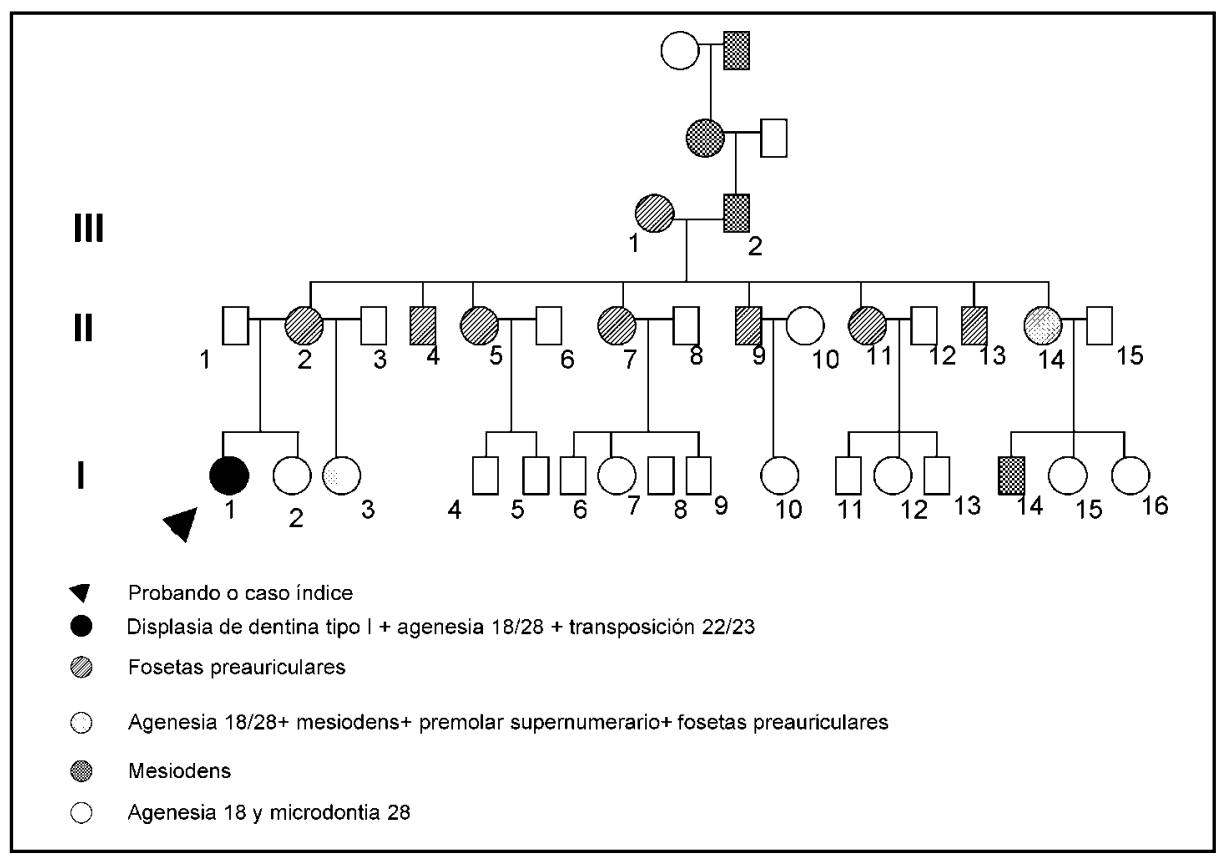

Figura 1. Genealogía de la familia estudiada 
Tabla 1. G enes candidatos estudiados

\begin{tabular}{|c|c|c|c|c|c|}
\hline Gen & $\begin{array}{l}\text { Mapeo } \\
\text { cromosómico }\end{array}$ & $\begin{array}{l}\text { Expresión } \\
\text { durante el } \\
\text { desarrollo } \\
\text { dental }\end{array}$ & $\begin{array}{l}\text { Ratones } \\
\text { knockout con } \\
\text { anomalías } \\
\text { dentarias }\end{array}$ & $\begin{array}{l}\text { Otra razón para } \\
\text { seleccionarlo como } \\
\text { gen candidato }\end{array}$ & Referencias \\
\hline $\begin{array}{l}\text { DSPP (dentin } \\
\text { sialophosphoprotein) }\end{array}$ & $4 \mathrm{q} 21.3$ & + & + & $\begin{array}{l}\text { Mutaciones en DSPP } \\
\text { originan dentinogénesis } \\
\text { imperfecta y displasia de } \\
\text { dentina tipo II }\end{array}$ & $5,6,10,11$ \\
\hline $\begin{array}{l}\text { IRF6 (interferon } \\
\text { regulatory factor 6) }\end{array}$ & 1q32.3-q41 & + & $\begin{array}{l}\text { no } \\
\text { disponible }\end{array}$ & $\begin{array}{l}\text { Mutaciones en IRF6 } \\
\text { originan síndrome Van } \\
\text { der Woude, que incluye } \\
\text { agenesia dental como } \\
\text { parte de su fenotipo }\end{array}$ & 12 \\
\hline $\begin{array}{l}\text { FGFR1 (fibroblast } \\
\text { growth factor } \\
\text { receptor 1) }\end{array}$ & $\begin{array}{l}\text { 8p11.2- } \\
\text { p11.1 }\end{array}$ & + & - & $\begin{array}{l}\text { Mutaciones en FGFR1 } \\
\text { originan síndrome de } \\
\text { Kallmann, que incluye } \\
\text { agenesia dental como } \\
\text { parte de su fenotipo }\end{array}$ & 13,14 \\
\hline $\begin{array}{l}\text { MSX1 (muscle } \\
\text { segment homeobox } \\
\text { homolog } 1 \text { - } \\
\text { Drosophila) }\end{array}$ & $4 \mathrm{p} 16$ & + & + & $\begin{array}{l}\text { MSX1 está asociado a } \\
\text { agenesia dental en } \\
\text { humanos }\end{array}$ & 15,16 \\
\hline $\begin{array}{l}\text { MSX2 (muscle } \\
\text { segment homeo box } \\
\text { homolog } 2 \text { - } \\
\text { Drosophila) }\end{array}$ & $5 q 34-q 35$ & + & + & - & 17 \\
\hline PAX9 (paired-box 9) & 14q12-q13 & + & + & $\begin{array}{l}\text { PAX9 está asociado a } \\
\text { agenesia dental en } \\
\text { humanos }\end{array}$ & $2,16,18,19$ \\
\hline $\begin{array}{l}\text { PRDM16 (PR-domain } \\
\text { zinc finger protein 16) }\end{array}$ & $\begin{array}{l}\text { 1p36.23- } \\
\text { p33 }\end{array}$ & + & $\begin{array}{l}\text { no } \\
\text { disponible }\end{array}$ & - & - \\
\hline $\begin{array}{l}\text { TGFA (transforming } \\
\text { growth factor alpha) }\end{array}$ & $2 p 13$ & + & - & $\begin{array}{l}\text { TGFA está asociado a } \\
\text { agenesia dental en } \\
\text { humanos }\end{array}$ & $16,20-23$ \\
\hline
\end{tabular}

dCTP, dGTP y dTTP); 1,5 mM MgCl2; 10 mM Tris/ HCL pH 8,3; $50 \mathrm{mM} \mathrm{KCL;} \mathrm{0,25-1,0} \mu \mathrm{M}$ de cada partidor; y 0,01-0,02 U de Taq polimerasa/ul. Los partidores para IRF6, FGFR1, MSX1, MSX2, PAX9 y TGFA fueron obtenidos de la literatura ${ }^{11,13-18}$. Los partidores para el gen DSPP se describen en la Tabla 2. La secuencia de los partidores para el gen
PRDM16 se obtuvo por gentileza del Dr. Bryan Bjork del Brigham and Women's Hospital de Harvard Medical School, Boston, USA.

Los ciclos de secuenciación fueron realizados en volúmenes de $20 \mu \mathrm{l}$ usando $4 \mu \mathrm{l}$ del reactivo determinante de secuencia ABI Big Dye, $1 \mu$ de 5 $\mu \mathrm{M}$ de partidor de secuenciación, $1 \mu \mathrm{l}$ de DMSO , 4 
Tabla 2. Partidores del gen D SPP

\begin{tabular}{|lll|}
\hline Gen & Exón & Partidores \\
& Exón 1rev & 5-TGGTTTGTTGAAACCTCACA-3 \\
& Exón 2brev & 5-TCAATGATCTTGAAGGAAAGCA-3 \\
& Exón 2\&3arev & 5-CCCATTCCAGCCCTCATT-3 \\
& Exón 3aBfor & 5-TTGATTGAATTGTTCTTTTCAG-3 \\
& Exón 3bfor & 5-GTGTCCTGGTGCATGAAGGT-3 \\
& Exón 3cfor & 5-GCCACAAACAGAAGCAACAC-3 \\
& Exón 3dfor & 5-GTCCTAGTGGGAATGGAGCA-3 \\
& Exón 4afor & 5-CCTATGGCAACTTTCCCAGT-3 \\
& Exón 4bfor & 5-TGACAGCAATAGTGATGGATATGA-3 \\
Exón 4cfor & 5-CAAATCAGACAGTGGCAAAGG-3 \\
& Exón 4dfor & 5-AGCAAGTCAGACAGCAGCAA-3 \\
& Exón 4efor & 5-AGCAATAGCAGTGAGAGCAGTG-3 \\
& Exón 4ffor & 5-AGCAATAGCAGTGAGAGCAGTG-3 \\
& Exón 4frev & 5-CTATTGCTGCTGTCGCTATCA-3 \\
\hline
\end{tabular}

$\mu \mathrm{l}$ de $2,5 \mathrm{X}$ buffer, y 2,5 $\mathrm{ng} / 100$ de pares de bases de ADN templado, los cuales fueron sometidos a un paso de denaturación a $96^{\circ} \mathrm{C}$ por $30 \mathrm{~s}$; las reacciones estuvieron en ciclos de secuenciación a $96^{\circ} \mathrm{C}$ por $10 \mathrm{~s}, 50^{\circ} \mathrm{C}$ por $5 \mathrm{~s}, \mathrm{y} 60^{\circ} \mathrm{C}$ por 4 min por 40 ciclos. La purificación fue realizada de acuerdo a protocolos estandarizados, las muestras fueron resuspendidas en 40 a $100 \mu \mathrm{l}$ de ddH2O, y 2,5 $\mu \mathrm{l}$ fueron inyectados sobre un secuenciador Applied Biosystems 3700. Se utilizó el software de secuenciación ABI (versión 2.1.2). Los cromatogramas fueron transferidos a una estación de trabajo Unix, denominada PHRED (versión 0.961028), ensamblada con PHRAP (versión 0.960731), el análisis se efectuó por POLYPHRED (versión 0.970312), y los resultados fueron vistos con el programa CONSED (versión 4.0) ${ }^{24}$. Cuando los resultados indicaban la posibilidad de una variante nueva, se secuenciaron y analizaron las muestras de otros miembros de la familia disponibles y controles poblacionales, utilizando el mismo método.

\section{RESULTADOS}

Se estudió a una familia con tres generaciones afectadas por anomalías dentarias en la rama materna, cuya genealogía es presentada en la Figura 1.
No se encontró mutaciones en los genes FGFR1, MSX2, PAX9, PRDM16 o TGFA en alguno de los integrantes de la familia.

Dentro del estudio del gen MSX1, se encontró una mutación sin sentido: G16D, presente tanto en personas afectadas, como no afectadas y dentro de las afectadas en personas con los tres fenotipos descritos (I-2, I-3, I-6, I-9, I-14, II-1, II-2, II-7, II-9, II-11, II-14 y III-2).

Al considerar el análisis del gen IRF6, se detectó la existencia de dos mutaciones en condición de homocigoto compuesto en el propósito (I1), con displasia de dentina tipo 1 , dentro de la región no codificante en el extremo $5^{\prime}$ del exón 1 con la siguiente localización: G224C y G246A, siendo los padres de la paciente heterocigotos para las dos mutaciones descritas (Figura 2).

Respecto al gen DSPP, se encontraron también dos mutaciones diferentes. Una de ellas es una mutación silente en el codón 299, A por G, codificándose en las dos situaciones una serina, sin interferir en la cadena proteica (II-14). La otra mutación se encontró igualmente en el exón 3 (G165R), produciéndose de esta manera un cambio de una glicina por una arginina en la cadena proteica. Esta última mutación se pesquisó en una paciente hasta el momento asintomática, con tan sólo 2 años de edad (I-15). 


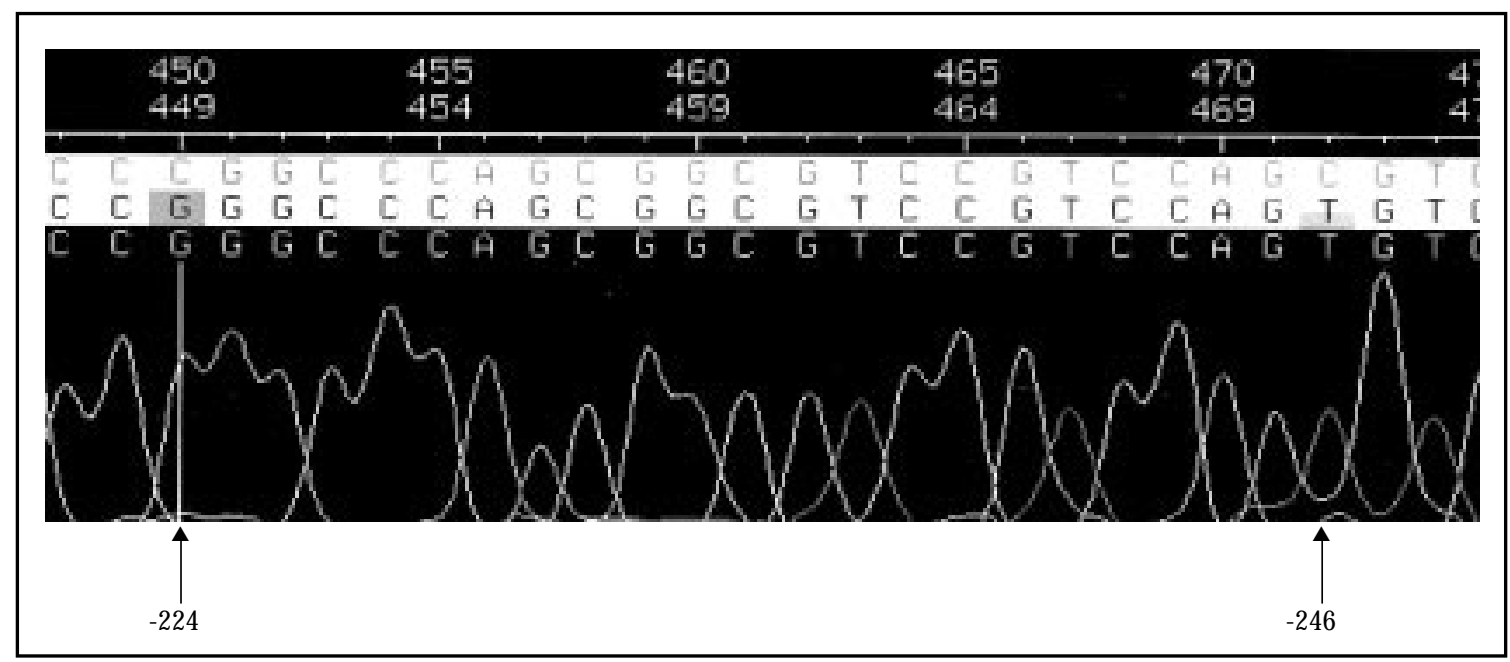

Figura 2. Secuencia de ADN de la probando (I-1) que evidencia dos mutaciones en el gen IRF6, dentro de la región no codificante en el extremo 5’ del exón 1 en las posiciones G224C y G246A.

\section{DisCUSIÓN}

Dentro del análisis de la familia expuesta, sólo se encontraron mutaciones en dos genes, MSX1 y DSPP.

No se encontró, sin embargo, una correlación genotipo-fenotipo con la mutación G16D del gen MSX1. Esta misma mutación ha sido descrita previamente en población chilena dentro de estudios de pacientes con fisura palatina, encontrándose en personas afectadas y no afectadas, de manera similar que en nuestro reporte actual. Se ha postulado que esta mutación podría interferir destruyendo una secuencia exónica incrementadora y creando una nueva para una proteína diferente rica en serina/arginina, $\operatorname{SRp} 40^{25}$.

Las mutaciones descritas en condición homocigota en la probando en el gen IRF6 no han sido descritas previamente. No pudimos determinar si estos cambios eran funcionales o no. Se piensa realizar a futuro estudios con análisis de modelos computacionales relacionados, estudios funcionales a través de ratones knockout o bien, efectuando estudios con grupos de pacientes con esta enfermedad en búsqueda de ésta u otras mutaciones en este gen que pudieran orientar sobre la relación causal de ésta en la displasia de dentina tipo 1.

El gen DSPP se consideró dentro del estudio como gen candidato para la etiología de la displasia de dentina tipo 1, debido a la descripción en artículos previos de otros autores de mutaciones en el exón 2 del gen DSPP en pacientes con dentinogénesis imperfecta y dado el mapeamiento en el cromosoma 4q21.3 de la displasia de dentina tipo 2 , región en la cual se encuentra el gen DSPP y porque codifica la disialofosfoproteína, la segunda proteína más frecuente de la matriz dentiniana ${ }^{8,9}$. Aunque en la propósito, con fenotipo compatible con DD tipo 1, no se encontraron mutaciones en los exones 1, 2, 3 ó 4 del gen DSPP, ello no descarta que no se encuentre implicado en la etiología de la enfermedad, dado que podrían existir mutaciones en el exón 5. No obstante, la amplificación de este exón es difícil de efectuar, experiencia que ya ha sido descrita por otros autores que han trabajado con el gen DSPP, condición que posiblemente esté dada por la cantidad de secuencias repetidas que porta el gen a este nivel ${ }^{26}$. Los templados ricos en repeticiones en dinucleótidos y tetranucleótidos y otras secuencias con repeticiones en tandem son difíciles de secuenciar adecuadamente por cualquier técnica individual disponible. Durante la secuenciación de una región altamente repetida, la tasa a la cual el nucleótido relevante marcado es consumido es muy alta y esto afecta su posterior incorporación en la secuenciación. Además, la enzima utilizada en la 
secuenciación está sujeta a una cierta cantidad de pérdida, condición que se vuelve crítica en una secuencia altamente repetida, donde son creados numerosos sitios de iniciación de copia de la secuencia, lo que origina que la enzima disminuya aún más al interactuar en varios sitios aleatorios en la región altamente repetida.

Otros genes que podrían ser considerados para estudiar dentro de la etiología de la displasia de dentina tipo 1 son aquellos mapeados cerca de DSPP como osteopondina (SPP1), proteína de la matriz de la dentina (DMP1), y sialoproteína ósea II (IBSP), o bien diferentes moléculas componentes de la matriz extracelular presentes durante el desarrollo dentario como: factores de crecimiento, proteínas derivadas del suero, lípidos y enzimas degradantes, como las proteinasas ${ }^{27-29}$.

Respecto a la segunda mutación detectada en el exón 3 del gen DSPP, y que induce un cambio de glicina por arginina, en una paciente asintomática (Figura 1, I-15), no puede aún interpretarse ni definirse un fenotipo dental consecuente dada la

\section{REFERENCIAS}

1. Scarel-Caminaga RM, Pasetto S, Ribeiro E, Peres RCR. Genes and tooth development: reviewing the structure and function of some key players. Braz J Oral Sci 2003; 2: 339-47.

2. Mostwska A, Biedziak B, Trzeciak WH. A novel mutation in PAX9 causes familial form of molar oligodontia. Eur J Hum Genet 2006; 14: 173-9.

3. Heliovara A, Ranta R, Rautio J. Dental abnormalities in permanent dentition in children with submucous cleft palate. Acta Odontol Scand 2004; 62: 129-31.

4. Yoshida $T$, Kanegane $H$, Yanagida $M$, Osato $M$, MiyaKaWi T, Ito Y et al. Functional analysis of RUNX2 mutations in Japanese patients with cleidocranial dysplasia demonstrates novel genotype-phenotype correlation. Am J Hum Genet 2002; 71: 724-38.

5. Xiao S, Yu C, Chou X, Yuan W, Wang Y, Bu L et al. Dentinogenesis imperfecta 1 with or without progressive hearing loss is associated with distinct mutations in DSPP. Nature Genet 2001; 27: 201-4.

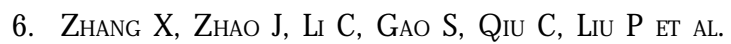
DSPP mutation in dentinogenesis imperfecta Shields type II. Nature Genet 2001; 27: 151-2. edad de 2 años de la niña, puesto que aún falta definir en ella la dentición permanente, se debiera seguir su evolución para observar si presenta alguna alteración clínica. Este punto nos demuestra, una vez más, que efectuar estudios moleculares en niños genera dificultades por las incertezas en la predicción de riesgos genéticos y constituye un dilema ético real.

Este trabajo es una experiencia pionera en el estudio de causas genéticas de anomalías dentarias en población chilena, en el cual se describe la presencia de una mutación no descrita en el gen IRF6 en una paciente con displasia de dentina tipo 1, hallazgo que debe complementarse con estudios bioinformáticos, funcionales y poblaciones extensos de pacientes afectados por la misma condición para su validación como agente etiológico. Además se resalta la presencia de una nueva mutación en el gen DSPP en una niña asintomática, lo cual ratifica los conflictos éticos y de consejería genética del estudio de pacientes menores o presintomáticos.

7. Rapjar H, Koch MJ, Davies RM, Melody KT, Kielty CM, Dixon MJ. Mutation of the signal peptide region of the bicistronic gene DSPP affects translocation to the endoplasmic reticulum and results in defective dentine biomineralization. Hum Mol Genet 2002; 11: 2559-65.

8. Shankiy PE, Mackie IC, Sloan P. Dentinal Dysplasia type I: Report of a case. Int J Paediat Dent 1999; 9: 37-42.

9. EFfrey D, James K, Hartsheid J, Wrigth JT, Hart TC. Dentine dysplasia, type II. Linkage to chromosome 4p. J Craniofac Genet Dev Biol 1997; 17: 171-7.

10. Sreenath T, Thyagarajan T, Hall B, Longenecker G, D'souza R, Hong S et al. Dentin sialophosphoprotein knockout mouse teeth display widened predentin zone and develop defective dentin mineralization similar to human dentinogenesis imperfecta type III. J Biol Chem 2003; 278: 24874-80.

11. Rajpar MH, Koch MJ, Davies RM, Melody KT, Kielty CM, Dixon MJ. Mutation of the signal peptide region of the bicistronic gene DSPP affects translocation to the endoplasmic reticulum and results in defective dentine biomineralization. Hum Mol Genet 2002; 11: 2559-65.

12. Kondo S, Schutte BC, Richardson RJ, Bjork BC, Knight AS, Waranabe $Y$ ET aL. Mutations in 
interferon regulatory factor 6 cause Van der Woude and Popliteal Pterygium syndromes. Nature Genet 2002; 32: 285-9.

13. Kettunen P, Karavanova I, Thesleff I. Responsiveness of developing dental tissues to fibroblast growth factors: expression of splicing alternatives of FGFR1, -2, -3, and of FGFR4; and stimulation of cell proliferation by FGF-2, $-4,-8$, and -9 . Dev Genet 1998; 22: 374-85.

14. Dode C, Leviliers J, Dupont JM, De Paepe A, Le Du $\mathrm{N}$, Soussi-Yanicostas N ET AL. Loss-of-function mutations in FGFR1 cause autosomal dominant Kallmann syndrome. Nature Genet 2003; 33: 463-5.

15. Satokata I, Maas R. Msx1 deficient mice exhibit cleft palate and abnormalities of craniofacial and tooth development. Nature Genet 1994; 6: 348-56.

16. Vieira AR, Meira R, Modesto A, MurRay JC. MSX1, PAX9, and TGFA contribute to tooth agenesis in humans. J Dent Res 2004; 83: 723-7.

17. Satokata I, Ma L, Ohshima H, Bei M, Woo I, NishizaWA K ET AL. Msx2 deficiency in mice causes pleiotropic defects in bone growth and ectodermal organ formation. Nature Genet 2000; 24: 391-5.

18. Peters H, Dol U, Niessing J. Differential expression of the chicken Pax-1 and Pax-9 gene: in situ hybridization and immunohistochemical analysis. Dev Dyn 1995; 203: 1-16.

19. VIEIRA AR. Oral clefts and syndromic forms of tooth agenesis as models for genetics of isolated tooth agenesis. J Dent Res 2003; 82: 162-5.

20. Vieira AR, Avila JR, Daack-Hirsch S, Dragan E, FélX TM, Rahimov F ET aL. Medical sequencing of candidate genes for nonsyndromic cleft lip and palate. PLoS Genet 2005; 1: e64.

21. Dixon MJ, Ferguson MWJ. The effects of epidermal growth factor, transforming growth factor $\alpha$ and $\beta$ and platelet-derived growth factor on murine palatal shelves in organ culture. Arch Oral Biol 1992; 37: 395-410.

22. Luetteke NC, Qiu TH, PeifFer RL, Oliver P, Smithies $\mathrm{O}$, LEE DC. TGF $\alpha$ deficiency results in hair follicle and eye abnormalities in targeted and waved-1 mice. Cell 1993; 73: 263-78.

23. Mann GB, Fowler KJ, Gabriel A, Nice EC, Wimams RL, DunN AR. Mice with a null mutation of the TGF $\alpha$ gene have abnormal skin architecture, wavy hair, and curly whiskers and often develop corneal inflammation. Cell 1993; 73: 249-61.

24. Nickerson DA, Tobe VO, TaYlor SL. PolyPhred: automating the detection and genotyping of single nucleotide substitutions using fluorescence-based resequencing. Nucleic Acids Res 1997; 25: 2745-51.

25. Vieira AR, Castilo S, Aravena T, Astete C, Sanz P, TASTETS ME ET AL. Análisis mutacional del gen Homeobox de segmento muscular 1 (MSX1) en chilenos con fisuras orales. Rev Méd Chile 2004; 132: 816-22.

26. Gu KI, Chang S, Ritchie H, Clarkson B, Rutherford B. Molecular cloning of a human dentin sialophosphoprotein gene. Eur J Oral Sci 2000; 108: 35-42.

27. Fena JQ, Huang H, Lu Y, Ye L, Xie Y, Tsutsui TW et AL. Dentin matrix protein 1 (Dmp 1) is specially expressed in mineralizad, but not soft, tissues during development. J Dent Res 2003; 82: 776-80.

28. Bègue-Kirn C, Krebsbach P, Bartlett J, Butler W. Dentin sialoprotein, dentin phosphoproteine, enamelysin and ameloblastin: tooth-specific molecules that are distinctively expressed during murine dental differentiation. Eur J Oral Sci 1998; 106: 963-70.

29. Pragna P. Soundbites. Nature Genet 2001; 27: 12930.

\section{Agradecimientos}

Los autores agradecen a la familia que entusiastamente participó de este proyecto, asimismo al Dr. Jeffrey C. Murray por su aporte durante todas las etapas de este estudio, además al Servicio de Salud Metropolitano Sur Oriente de Chile, al Instituto para la Nutrición y Tecnología de Alimentos (INTA) y al Hospital Clínico Universidad de Chile por su contribución para la realización de este trabajo. 